\title{
ARTICLE
}

Received 18 Sep 2013 | Accepted 22 Nov 2013 | Published 3 Jan 2014 DOl: 10.1038/ncomms3992

\section{Tuning the electron transport at single donors in zinc oxide with a scanning tunnelling microscope}

Hao Zheng ${ }^{1}$, Alexander Weismann ${ }^{1} \&$ Richard Berndt ${ }^{1}$

In devices like the single-electron transistor the detailed transport properties of a nanostructure can be measured by tuning its energy levels with a gate voltage. The scanning tunnelling microscope in contrast usually lacks such a gate electrode. Here we demonstrate tuning of the levels of a donor in a scanning tunnelling microscope without a third electrode. The potential and the position of the tip are used to locally control band bending. Conductance maps in this parameter space reveal Coulomb diamonds known from three-terminal data from single-electron transistors and provide information on charging transitions, binding energies and vibrational excitations. The analogy to single-electron transistor data suggests a new way of extracting these key quantities without making any assumptions about the unknown shape of the scanning tunnelling microscope tip.

\footnotetext{
${ }^{1}$ Institut für Experimentelle und Angewandte Physik, Christian-Albrechts-Universität zu Kiel, D-24098 Kiel, Germany. Correspondence and requests for materials should be addressed to A.W. (email: weismann@physik.uni-kiel.de).
} 
A s the size of semiconductor devices decreases, individual dopants become decisive for the performance of devices ${ }^{1,2}$. Moreover, $\operatorname{spin}^{3}$ or charge ${ }^{4}$ states of single dopants have been proposed as key components of a solid-state quantum computer. The scanning tunnelling microscope (STM) has been used to characterize defect states of single dopants ${ }^{2}$ and to manipulate their position and charge state ${ }^{5-8}$. There are predictions that double donors, which can deliver two electrons, may be particularly useful for addressing spin states in quantum computers ${ }^{9,10}$.

In artificial atoms, as realized in a single-electron transistor (SET), it is possible to tune the energies of electronic states using a gate electrode. In contrast to single atoms on metal surfaces, whose ground state is determined by the impuritysubstrate combination, transport experiments at different gate voltages can explore a wider parameter space. Signatures of single donors have been resolved and valuable information such as the electron-binding energy have been extracted ${ }^{11-14}$. Being a two-terminal device STM lacks a gate electrode for tuning the energy levels of nanostructures. To avoid this limitation, lithography has been used to fabricate a back gate ${ }^{15,16}$ or source and drain contacts with the STM tip acting as a moveable gate ${ }^{17-19}$.

Here we present an experimental approach to tune the levels of single quantum defects without a third electrode. It relies on the local band bending induced by the STM tip, which may be controlled by two independent parameters, the tip position and the sample voltage. Maps of the differential conductance in this parameter space closely resemble the Coulomb diamonds known from three-terminal experiments on $\mathrm{SETs}^{20}$ and provide information on charge transitions, binding energies and vibrational excitations. We analyse double donors-most likely $\mathrm{Zn}$ interstitials ${ }^{21}$-close to the $\mathrm{ZnO}(0001)$ surface by measuring the differential conductance $d I / d V$ versus lateral position $x$ and sample voltage $V$, and show that a conventional two-terminal STM may be used to acquire SET-like data.

\section{Results}

Tip-induced band bending. Nominally, undoped $\mathrm{ZnO}$ exhibits $n$-type conductivity, presumably due to intrinsic defects like $\mathrm{Zn}$ interstitials ${ }^{21,22}$. On our samples, we observed donor densities of $\approx 0.7 \ldots 1.5 \times 10^{12} \mathrm{~cm}^{-2}$. When the Au tip is brought close to an $n$-type $\mathrm{ZnO}$ crystal at zero bias voltage, the work function difference between the two materials causes the energy bands of $\mathrm{ZnO}$ to bend upwards. A positive (negative) voltage applied to the sample can increase (decrease) the band bending. Thus, the work function difference and the bias voltage result in a tip-induced band bending (TIBB). Its lateral extension depends on the tip radius of curvature and the tipsample distance along with material parameters. Consequently, the applied voltage does not drop entirely inside the vacuum barrier but also inside the semiconductor and the chemical potential varies with depth and lateral distance to the STM tip. TIBB complicates the interpretation of $d I / d V$ spectroscopy data because it shifts spectroscopic features. Moreover, it may move states across the Fermi energy of the semiconductor, thus changing their occupation and inducing additional spectral features.

Differential conductance spectra. Figure 1 shows typical examples of differential conductance spectra from three classes of donors that correspond to different occupations with electrons at zero bias. The pronounced features can be attributed to donor states, charge transitions and vibronic excitations. The assignment of the observed signatures to specific processes and the identification of the charge state will become obvious below, when the lateral position $x$ of the tip will be used as an additional parameter. The defect level of the donor of Fig. 1a is doubly occupied and the donor thus is neutral at zero bias ${ }^{21}$. The STM tip can extract electrons from the donor state resulting in a peak at a negative sample voltage (marked $D^{0}$ ). In addition, an equidistant sequence of vibronic excitations (vertical bars) is observed. The peak at positive sample bias (marked $0 /+$ ) corresponds to the voltage required to shift the donor state above the Fermi energy of the sample, which changes the charge of the donor from neutral to $+e$. Such ionization signatures in $d I / d V$ spectra have also been observed on subsurface dopants in other semiconductors ${ }^{6,23}$, topological insulators ${ }^{24}$ and molecular thin films ${ }^{25}$, as well as on adatoms on graphene ${ }^{26}$. The donor of Fig. $1 \mathrm{~b}$ is singly occupied at zero bias. Thus, the STM tip can add or remove an electron at positive and negative voltages, respectively. Correspondingly, the 'donor level' appears twice in the spectrum as peaks $D^{0}$ and $D^{+}$surrounding the Fermi energy $E_{\mathrm{F}}$. The splitting between these features is related to the Coulomb repulsion between two electrons in the donor level and will be discussed below in conjunction with the Coulomb blockade regime of the SET. In addition, two ionization peaks are observed (marked by $0 /+$ and $+/++$ ) that indicate the transitions from the singly charged to the neutral and doubly charged configurations, respectively. In Fig. 1c, the donor level is empty at zero bias. Thus, electrons can be added by the STM tip resulting in a peak (marked $\mathrm{D}^{+}$) above $E_{\mathrm{F}}$ along with vibronic transitions. A negative bias voltage can pull the defect level below $E_{\mathrm{F}}$ of the sample resulting in a singly occupied state and a sharp peak in the $d I / d V$ spectrum.

Differential conductance maps. To further confirm the peak assignment, we measured series of $d I / d V$ spectra at densely spaced positions along straight lines crossing the buried donors. These data are shown as two-dimensional colour-coded maps in Fig. 1d-f. The maps display one (Fig. 1d) or two (Fig. 1e,f) conductance maxima that appear as approximate parabolae (dash dotted lines). These parabolae are due to the ionization peaks introduced in Fig. 1a-c and are labelled accordingly. The variation of the ionization peaks with lateral position is due to the change of the TIBB at the position of the donor. To pull an occupied level above $E_{\mathrm{F}}$, a small positive voltage is required if the donor is right below the tip $\left(D^{0}\right.$ in Fig. $1 \mathrm{~d}$, and $\mathrm{D}^{+}$in Fig. 1e). This voltage is increased at larger separations, which gives rise to the parabolae observed at $V>0$ in Fig. 1d,e. The parabolae at negative sample bias are due to empty levels that are pushed below $E_{\mathrm{F}}\left(D^{0}\right.$ in Fig. 1e and $\mathrm{D}^{+}$in Fig. 1f). This requires the contact potential, which is maximal below the tip, be compensated. The voltage $|V|$ for ionization therefore is largest for a donor below the tip apex ${ }^{27}$. The variation of the donor levels $D^{0}$ and $D^{+}$and the vibrational excitations with lateral position reflects the TIBB in the semiconductor.

Data analysis. In previous modelling of STM measurements on subsurface dopants, the Poisson equation was solved to determine the TIBB ${ }^{6,23}$. This involves a number of parameters. While some of them, for example, the band gap and the dielectric constant, may be taken from literature, others such as the tip-sample separation, the radius of curvature of the tip and its shank angle are a priori unknown and have to be adjusted to match the experimental data. In our approach, the donor is modelled by a single orbital with an energy $\varepsilon_{\mathrm{d}}$ (defined with respect to the local chemical potential $\mu_{\mathrm{c}}$ ) and a Coulomb repulsion $U$ in the case of double occupancy. The total energies $E_{n}$ for occupation numbers 
a

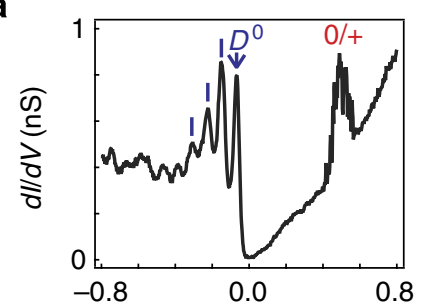

d

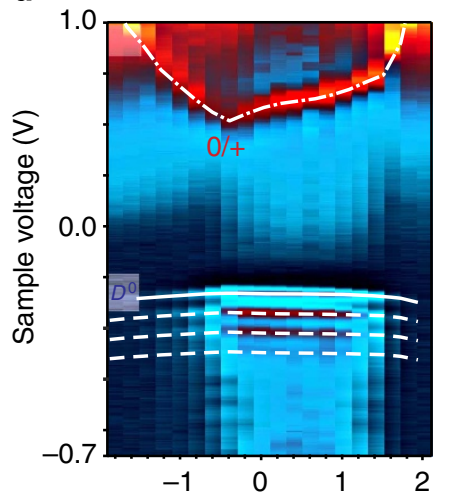

b

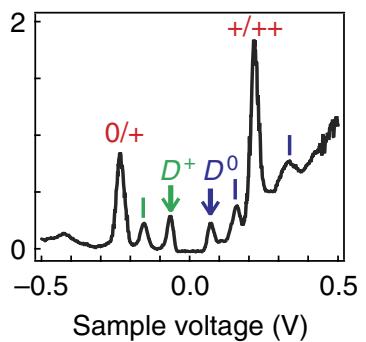

e

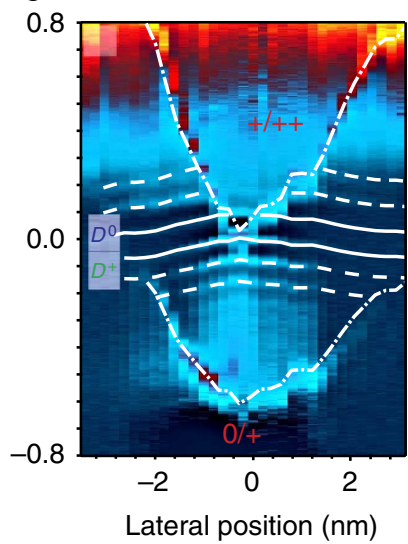

C

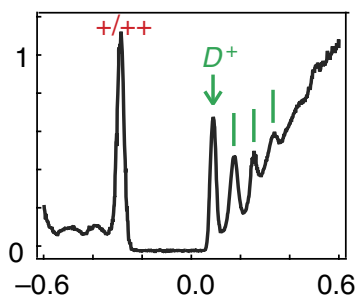

f

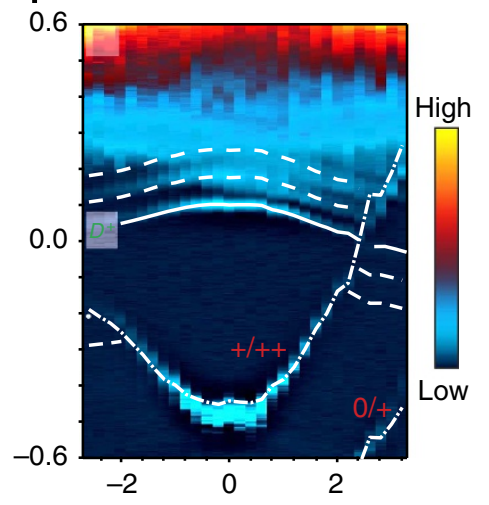

Figure 1 | Spectra of the differential conductance (dI/dV) of donors in ZnO. At zero bias, the donors are (a) neutral, (b) singly charged and (c) doubly charged. $D^{0}$ and $D^{+}$indicate the levels of the donors, $\mathrm{O} /+$ and $+/++$ mark ionization peaks. At voltages below (above) $0 /+$ the donor is neutral (singly charged). $+/++$ marks transitions between the singly and the doubly charged states. Vertical bars indicate resolved vibrational features. Tunnelling parameters before disabling current feedback: $I=3.0 \mathrm{nA}, V=1.0,0.5$ and $0.6 \mathrm{~V}$, respectively. Panels $\mathbf{d}$-f show spatially resolved colour-coded $d l / d V(x, V)$ maps from donors that are (d) neutral, (e) singly charged and (f) doubly charged at zero bias. Before disabling current feedback, the STM was operated at $I=3 \mathrm{nA}$ and sample voltages of 1.0, 0.8 and $0.6 \mathrm{~V}$, respectively. The colour range corresponds to conductances of 0-1.2, $-0.2-1.4$ and 0-1.5 $\mathrm{nS}$. Scans were performed along a line oriented $13^{\circ}$ with respect to a $\langle 11 \overline{2} 0\rangle$ direction. Simulation results are shown with lines. Dash dotted, solid and dashed lines indicate ionization lines $(0 /+$ and $+/++)$, defect levels $\left(D^{0}\right.$ and $\left.D^{+}\right)$and vibrational satellites, respectively. While spectra like in $\mathbf{d}$ were observed from isolated donors, e,f were typically found from closely spaced donors. In (d) the vibrational energy $\hbar \omega_{+} \approx 70 \mathrm{meV}$ is essentially independent of the tip-donor distance and similar values were measured above other isolated donors.

$n=0,1$ and 2 are

$$
E_{0}=0 \quad E_{1}=\mu_{\mathrm{c}}+\varepsilon_{\mathrm{d}} \quad E_{2}=2\left(\mu_{\mathrm{c}}+\varepsilon_{\mathrm{d}}\right)+U
$$

and correspond to the doubly charged, singly charged and neutral configurations, respectively.

For sample voltages $V$ below the inversion threshold and above flat band conditions, no charge accumulation occurs at the surface and the TIBB may be assumed to vary linearly with the bias voltage ${ }^{28}$.

$$
\mu_{\mathrm{c}}(x, V)=\beta(x) \cdot e\left(V-V_{\mathrm{F}}\right) .
$$

This leaves the factor $\beta(x)$ and the flat band voltage $V_{\mathrm{F}}$ as parameters to be extracted from the experimental data. $\beta(x)$ resembles the shape of the TIBB as a function of lateral distance $x$ and in a fixed depth inside the semiconductor. To calculate the illustrative examples in Fig. 2 and Supplementary Fig. S1, we used an analytical expression for $\beta$ that models the lateral variation of the TIBB at a given depth by a squared Lorentzian of width $\sigma$ :

$$
\beta(x)=\beta_{0}\left[\frac{\sigma^{2}}{\sigma^{2}+x^{2}}\right]^{2}
$$

The ionization voltages $V_{+/+}$and $V_{0 /+}$ fulfil the conditions $E_{0}=E_{1}$ and $E_{1}=E_{2}$, respectively, and separate ground states with different occupation numbers. They are given by

$$
e V_{+/++}(x)=\frac{\beta(x) e V_{\mathrm{F}}-\varepsilon_{\mathrm{d}}}{\beta(x)}
$$

and

$$
e V_{0 /+}(x)=\frac{\beta(x) e V_{\mathrm{F}}-\varepsilon_{\mathrm{d}}-U}{\beta(x)} .
$$

Since these conditions can always be met, two ionization parabolae (red curves in Fig. 2) are present in every $d I / d V$ map.

The data furthermore provide an opportunity to determine vibrational energies $\hbar \omega_{n}$ of a donor in different charge states. For instance, in Fig. 1d, an electron is extracted from a neutral donor at $V<0$. The vibronic features therefore reflect the vibrational energy of a singly charged donor ${ }^{29,30}$. In contrast, Fig. 1e shows the vibrations of a donor in its neutral and doubly charged states for $V>0$ and $V<0$, respectively. In our model, we allow the vibration energies $\hbar \omega_{n}$ to depend on the occupation number $n$ and hence on the charge of the donor. For positive $V$ direct tunnelling into the donor state increases $n$ by one $(n \rightarrow n+1)$ and $m$ vibronic modes of energy $\hbar \omega_{n+1}$ may be excited. This excitation requires an energy $e V^{>}=E_{n+1}-E_{n}+m \hbar \omega_{n+1}$. Electron extraction at negative $V$ decreases the occupation $(n \rightarrow$ $n-1)$ while exciting $m$ vibrations in charge state $n-1$. The required energy is $-e V^{<}=E_{n-1}-E_{n}+m \hbar \omega_{n-1}$. 

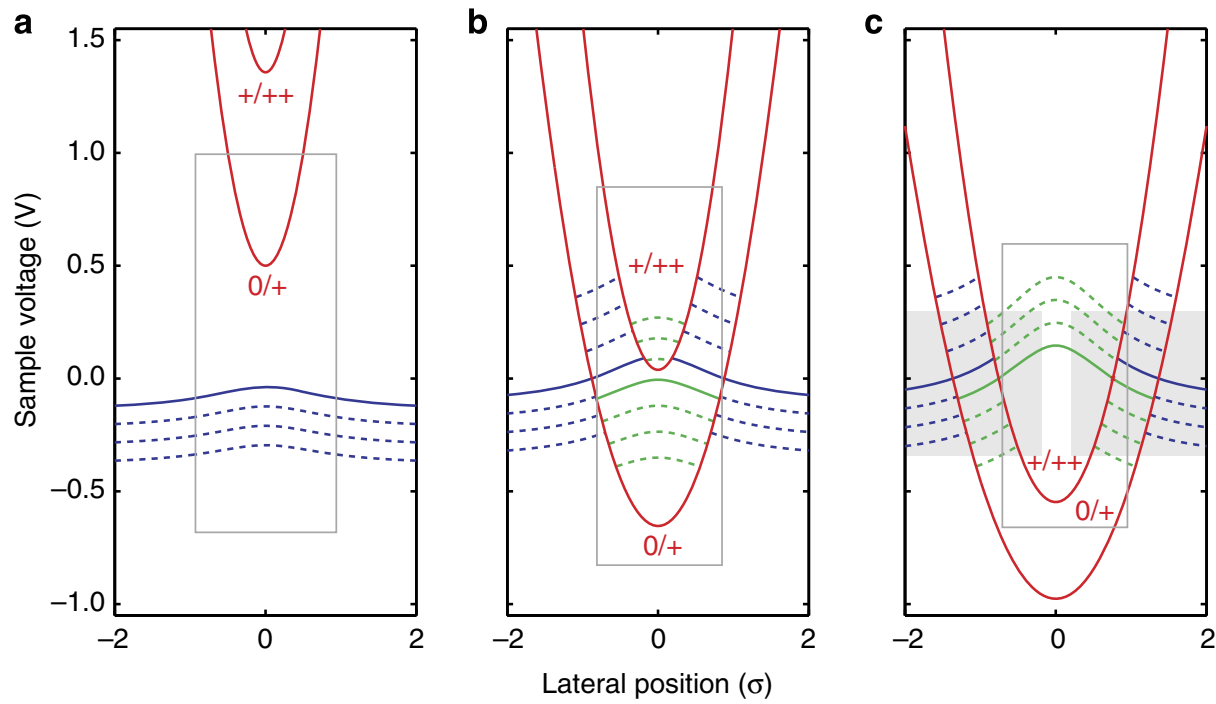

Figure 2 | Calculated energy diagrams. Calculated energy diagrams over a wide range of voltages and distances for a symmetric model tip where $\beta(x)$ is assumed to be a squared Lorentzian of width $\sigma$. Parameters in a-c were adjusted to approximate the data shown in Fig. 1d-f, respectively, which correspond to the areas marked by grey rectangles. Red curves represent the ionization lines. Blue and green solid lines are the donor levels of $D^{0}$ and $D^{+}$, dashed lines display the vibrational satellites. The shaded area in c exhibits a striking similarity to Coulomb blockade diamonds.

From the above equations we derive the following:

$$
\begin{gathered}
e V_{\mathrm{D}^{0}, m}^{<}(x)=\frac{-\beta(x) e V_{\mathrm{F}}+\varepsilon_{\mathrm{d}}+U-m \hbar \omega_{+}}{1-\beta(x)} \\
e V_{\mathrm{D}^{0}, m}^{>}(x)=\frac{-\beta(x) e V_{\mathrm{F}}+\varepsilon_{\mathrm{d}}+U+m \hbar \omega_{0}}{1-\beta(x)} \\
e V_{\mathrm{D}^{+}, m}^{<}(x)=\frac{-\beta(x) e V_{\mathrm{F}}+\varepsilon_{\mathrm{d}}-m \hbar \omega_{++}}{1-\beta(x)} \\
e V_{\mathrm{D}^{+}, m}^{>}(x)=\frac{-\beta(x) e V_{\mathrm{F}}+\varepsilon_{\mathrm{d}}+m \hbar \omega_{+}}{1-\beta(x)}
\end{gathered}
$$

For the elastic transitions $(m=0)$, we simplify the terminology of the above expressions to $V_{\mathrm{D}^{0}, 0}^{<}=V_{\mathrm{D}^{0} .0}^{>}=V_{\mathrm{D}^{0}}$ and $V_{\mathrm{D}^{+}, 0}^{<}=V_{\mathrm{D}^{+}, 0}^{>}=V_{\mathrm{D}^{+}}$. These expressions define the bias voltages of all spectroscopic features depicted in Fig. 2, namely, ionization lines $V_{+/++}$and $V_{0 /+}$ (red), donor levels $V_{\mathrm{D}^{0}}$ and $V_{\mathrm{D}^{+}}$(solid blue and green) and vibrational satellites (dashed blue and green).

We have applied the model to different kinds of donors (Supplementary Note 1 and Supplementary Table S1). An analysis of a number of donors reveals that the vibrational energy depends on the charge state (Supplementary Note 2 and Supplementary Fig. S2). The extracted binding energies, Coulomb repulsions and vibrational energies in different charge states are displayed in Table 1 and the Supplementary Table S2.

\section{Discussion}

A simplified presentation of the spectroscopic features of Fig. 1 is obtained by performing calculations for a symmetric tip and neglecting the local environment of the donors. Figure $2 \mathrm{a}$ and $b$ correspond to the cases of neutral and singly charged donors of Fig. 1d and e, respectively. Figure $2 c$ displays the most interesting case. In the shaded area, the intersections of the donor levels $D^{0}$ and $D^{+}$and the ionization lines $0 /+$ and $+I++$ are the four corners of a diamond-shaped area in the $x-V$ plane where the differential conductance vanishes because of Coulomb blockade.

In a SET (Fig. 3a), the energies of states and their occupations can be controlled by a gate voltage $V_{\mathrm{G}}$ while the current is driven between source and drain contacts using a voltage $V_{\mathrm{SD}}$. The differential conductance of a SET may be schematically described by the diagram in Fig. 3c. Lines with negative (positive) slope indicate conductance peaks that occur when the donor levels are in resonance with the Fermi level of the source (drain) contact. $n=0,1$ or 2 indicates the occupation numbers of the nanostructure at $V_{\mathrm{SD}}=0$. In the shaded areas Coulomb blockade occurs. The $d I / d V(x, V)$ map of a donor under a STM tip (Fig. 3d) is closely related. While the sample voltage $V$ corresponds to $V_{\mathrm{SD}}$ in a SET, the tip position $x$ plays the role of $V_{\mathrm{G}}$. In the experiments it was difficult to obtain a $d I / d V$ map with all $n=0$, 1,2 regions from a single donor. Therefore, Fig. $3 \mathrm{e}$ and g show data from two different donors, which exhibit occupations $n=0$, 1 and $n=1,2$, respectively. The schematics in Fig. 3 f,h label the main spectral features.

The analogy between STM and SET suggests a route to determine the Coulomb repulsion $U$ (Fig. $3 c, d$ ). It may be read off directly from the intersections of the conductance features $D^{0}$ and $(+/++)$ (dashed vertical lines). At these voltages, $\epsilon_{\mathrm{d}}+U$ and $\epsilon_{\mathrm{d}}$ are aligned with the Fermi levels of the tip and of $\mathrm{ZnO}$, respectively. In the same way, $D^{+}$and $(0 /+)$ intersect at $-U$.

In summary, we used a conventional two-terminal STM to perform measurements that are comparable to those in a three-terminal SET. $d I / d V(x, V)$ maps reveal that donors may be classified according to their charge states at zero bias. The donors display Coulomb blockade and charge-statedependent vibrational energies. They are analysed by a new model that is inspired by the analogy to a SET. The method may also be useful for other nanostructures buried in a semiconductor matrix. It may become possible to observe the influence of their charge state on the Kondo effect, metal-insulator transitions, or Franck-Condon blockade, similar to observations made from $\mathrm{SET}^{20,31}$ but on a much smaller length scale. 
Table 1 | Microscopic parameters (in meV) obtained from five donors.

\begin{tabular}{|c|c|c|c|c|c|c|}
\hline Fig. If & -208 & 80 & - & - & $61(9)$ & $69(1)$ \\
\hline Supplementary Fig. S2d & -336 & 99 & $49(6)$ & $66(3)$ & $63(2)$ & $64(6)$ \\
\hline Supplementary Fig. S2f & -416 & 99 & $55(6)$ & $63(3)$ & $66(12)$ & $66(2)$ \\
\hline
\end{tabular}

$\varepsilon_{\mathrm{d}}$ and $U$ are the binding energy and Coulomb repulsion. $\hbar \omega_{0}$ and $\hbar \omega_{++}$are the vibrational energies of neutral and doubly charged donor. $\hbar \omega_{+}^{<}$and $\hbar \omega_{+}^{>}$are the vibrational energies of singly charged donor determined from different parts of $d l / d V$ maps (cf. Supplementary Note 1 for a detailed definition). The uncertainties shown inside the brackets are the standard deviation of values extracted at different lateral distances of the tip from the donor.

a

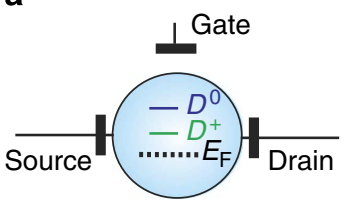

C
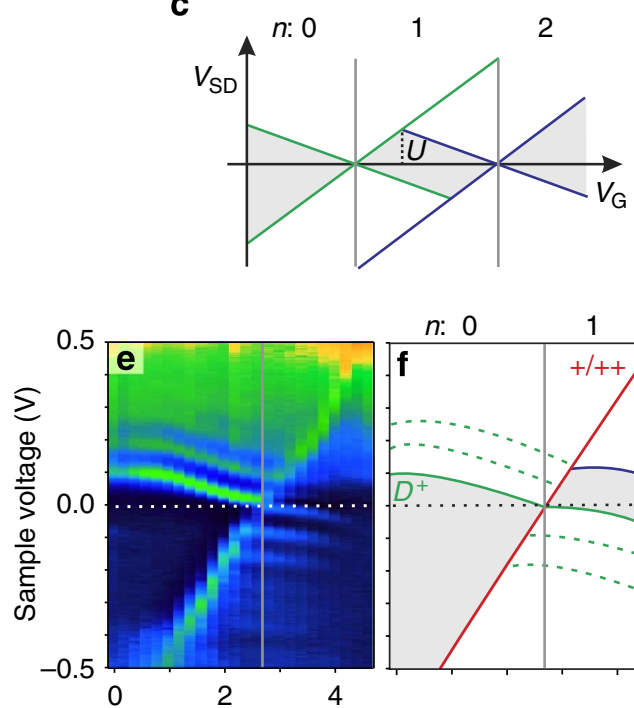

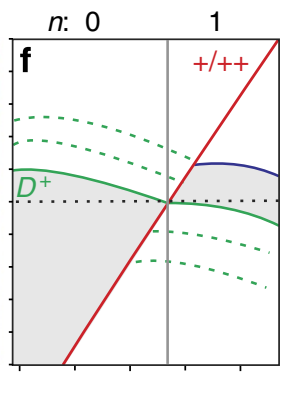

Lateral position $x(\mathrm{~nm})$ b

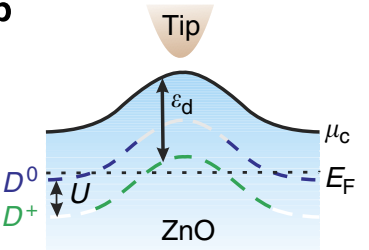

d

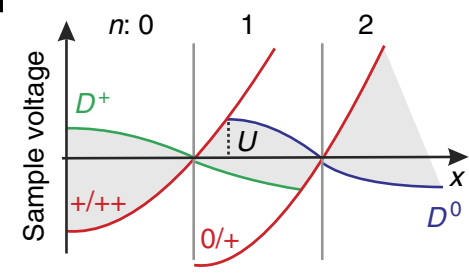

Figure 3 | Analogy between a donor in a SET and underneath a STM tip. (a) Schematics of a SET. Current is passed between the source and drain contacts via two localized levels $D^{0}$ and $D^{+}$, which can be shifted by applied voltage to the insulated gate contact. In the indicated case the donor is doubly charged. (b) Schematic diagram of local band bending in a semiconductor underneath a STM tip. $\mu_{\mathrm{c}}$, local chemical potential, $\epsilon_{\mathrm{d}}$, binding energy of the first electron in the donor level. The levels $D^{0}$ and $D^{+}$are marked by blue and green dashed lines when accessible by single-particle transitions and are coloured white otherwise. (c) Schematic two-dimensional map of conductance maxima of the SET versus source-drain voltage $V_{S D}$ and gate voltage $V_{G}$. $n$ is the occupation number. Depending on $V_{G}$ the number of electrons in the donor changes from $n=0$ over 1 to 2 . (d) Variation of the donor levels $D^{0}$ and $D^{+}$(blue and green solid lines) and the ionization lines $(0 /+)$ and $(+/++)$ (red lines) with distance $x$ from the tip apex and sample voltage $V$. In $\mathbf{c}$ and $\mathbf{d}$ vertical dotted lines indicate the Coulomb energy $U$. (e,g) $d l / d V(x, V)$ maps recorded along straight lines crossing buried donors. Before disabling current feedback, the STM was operated at $0.5 \mathrm{~V}, 3 \mathrm{nA}$. The colour bar represent $0-2 \mathrm{nS}$ in both maps. Panels $\mathbf{f}$ and $\mathbf{h}$ sketch the main conductance maxima from $\mathbf{e}$ and $\mathbf{g}$. Dotted lines indicate vibrational satellites. Yellow lines in $\mathbf{h}$ mark features that are attributed to nearby defects. In $\mathbf{c}, \mathbf{d}, \mathbf{f}$ and $\mathbf{h}$, shaded areas show Coulomb blockade.

\section{Methods}

Experimental details. All experiments were performed with a home-built STM operated at $5 \mathrm{~K}$ in ultra-high vacuum. Wurtzite $\mathrm{ZnO}(0001)$ single crystals were cleaned by $\mathrm{Ar}^{+}$bombardment and high temperature annealing. Imaging was performed at constant current with Au tips and the bias voltage was applied to the sample. Ex situ cut tips were gently brought into mechanical contact to the $\mathrm{ZnO}$ surface and retracted again. This procedure typically lead to the deposition of a $\mathrm{Au}$ cluster and often improved the sharpness of the tips. Spectra of $d I / d V$ were measured with a lock-in amplifier using a sinusoidal modulation of the bias with amplitudes of $10-15 \mathrm{mV}_{\text {rms }}$.
Analytical model. For quantitative analysis of the energies of the spectral features, the applied sample voltage must be converted into a chemical potential at the position of the donor. We thus define a scaling factor $\beta(x)$ to describe the change of the chemical potential $\mu_{\mathrm{c}}(x, V)=\beta(x) e\left(V-V_{F}\right)$ due to the voltage $V . V_{F}$ is the flat band voltage and $\beta(x)$ is a tip-specific function that resembles the shape of the TIBB as a function of lateral distance $x$. The donor is described by a single level with energy $\epsilon_{\mathrm{d}}$ with respect to the local chemical potential and an on-site Coulomb repulsion $U$ between two electrons in the localized state. $\beta(x), V_{F}, \epsilon_{\mathrm{d}}$ and $U$ are then determined by fitting the calculated energies to the experimental peak positions. Usually, retrieving such quantitative information from experiments is complicated 
by the unknown geometry of the tunnelling junction, which affects the TIBB. The present approach circumvents this problem as the tip-shape influences $\beta(x)$, which is extracted from the experimental data. We also compared the result with calculation using Poisson equations ( Supplementary Fig. 3 and Supplementary Note 3).

\section{References}

1. Roy, S. \& Asenov, A. Where do the dopants go? Science 309, 388-390 (2005).

2. Koenraad, P. M. \& Flatté, M. E. Single dopants in semiconductors. Nat. Mater. 10, 91-100 (2011)

3. Kane, B. E. A silicon-based nuclear spin quantum computer. Nature 393, 133-137 (1998).

4. Hollenberg, L. C. L. et al. Charge-based quantum computing using single donors in semiconductors. Phys. Rev. B 69, 113301 (2004).

5. Kitchen, D., Richardella, A., Tang, J., Flatte', M. E. \& Yazdani, A. Atom-byatom substitution of $\mathrm{Mn}$ in GaAs and visualization of their hole-mediated interactions. Nature 442, 436-439 (2006).

6. Teichmann, K. et al. Controlled charge switching on a single donor with a scanning tunneling microscope. Phys. Rev. Lett. 101, 076103 (2008)

7. Lee, D. H. \& Gupta, G. A. Tunable field control over the binding energy of single dopants by a charged vacancy in GaAs. Science 330, 1807-1810 (2010).

8. Zheng, H., Weismann, A. \& Berndt, R. Manipulation of subsurface donors in ZnO. Phys. Rev. Lett. 110, 226101 (2013).

9. Kane, B. E. et al. Single-spin measurement using single-electron transistors to probe two-electron systems. Phys. Rev. B 61, 2961-2972 (2000).

10. Calderon, M. J., Koiller, B. \& Sarma, S. D. Proposal for electron spin relaxation measurements using double-donor excited states in $\mathrm{Si}$ quantum computer architectures. Phys. Rev. B 75, 161304(R) (2007).

11. Sellier, H. et al. Transport spectroscopy of a single dopant in a gated silicon nanowire. Phys. Rev. Lett. 97, 206805 (2006).

12. Pierre, M. et al. Single-donor ionization energies in a nanoscale CMOS channel. Nat. Nanotech. 5, 133-137 (2010).

13. Fuechsle, M. et al. A single-atom transistor. Nat. Nanotech. 7, 242-246 (2012).

14. Lansbergen, G. P. et al. Gate-induced quantum-confinement transition of a single dopant atom in a silicon FinFET. Nat. Phys. 4, 656-661 (2008).

15. Zhang, Y. et al. Giant phonon-induced conductance in scanning tunnelling spectroscopy of gate-tunable graphene. Nat. Phys. 4, 627-630 (2008).

16. Deshpande, A., Bao, W., Zhao, Z., Lau, C. N. \& LeRoy, B. J. Imaging charge density fluctuations in graphene using Coulomb blockade spectroscopy. Phys. Rev. B 83, 155409 (2011).

17. Topinka, M. A. et al. Imaging coherent electron flow from a quantum point contact.. Science 289, 2323-2326 (2000).

18. Bockrath, M. et al. Resonant electron scattering by defects in single-walled carbon nanotubes. Science 291, 283-285 (2001)

19. Pioda, A. et al. Spatially resolved manipulation of single electrons in quantum dots using a scanned probe. Phys. Rev. Lett. 93, 216801 (2004).

20. Kastner, M. A. The single-electron transistor. Rev. Mod. Phys. 64, 849-858 (1992).
21. Zheng, H., Kröger, J. \& Berndt, R. Spectroscopy of single donors at $\mathrm{ZnO}(0001)$ surfaces. Phys. Rev. Lett. 108, 076801 (2012).

22. Look, D. C., Hemsky, J. W. \& Sizelove, J. R. Residual native shallow donor in ZnO. Phys. Rev. Lett. 82, 2552-2555 (1999).

23. Marczinowski, F. et al. Local electronic structure near Mn acceptors in InAs: surface-induced symmetry breaking and coupling to host states. Phys. Rev. Lett. 99, 157202 (2007)

24. Song, C.-L. et al. Gating the charge state of single Fe dopants in the topological insulator $\mathrm{Bi}_{2} \mathrm{Se}_{3}$ with a scanning tunneling microscope. Phys. Rev. B 86, 045441 (2012).

25. Pradhan, N. A., Liu, N., Silien, C. \& Ho, W. Atomic scale conductance induced by single impurity charging. Phys. Rev. Lett. 94, 076801 (2005).

26. Brar, V. W. et al. Gate-controlled ionization and screening of cobalt adatoms on a graphene surface. Nat. Phys. 7, 43-47 (2011).

27. Wijnheijmer, A. P. et al. Single Si dopants in GaAs by scanning tunneling microscopy and spectroscopy. Phys. Rev. B 84, 125310 (2011).

28. Loth, S., Wenderoth, M., Ulbrich, R. G., Malzer, S. \& Döhler, G. H. Connection of anisotropic conductivity to tip-induced space-charge layers in scanning tunneling spectroscopy of p-doped GaAs. Phys. Rev. B 76, 235318 (2007).

29. Nazin, G. V., Wu, S. W. \& Ho, W. Tunneling rates in electron transport through double-barrier molecular junctions in a scanning tunneling microscope. Proc. Natl Acad. Sci. USA 102, 8832-8837 (2005).

30. Nazin, G. V., Qiu, X. H. \& Ho, W. Vibrational spectroscopy of individual doping centers in a monolayer organic crystal. J. Chem. Phys. 122, 188105 (2005).

31. Leturcq, R. et al. Franck-Condon blockade in suspended carbon nanotube quantum dots. Nat. Phys. 5, 327-331 (2009).

\section{Acknowledgements}

This study was financially supported by the Deutsche Forschungsgemeinschaft through SFB 855 .

\section{Author contributions}

H.Z. and R.B. conceived the experiments, which were performed by H.Z. A.W. did the modelling. All authors discussed the data and prepared the manuscript.

\section{Additional information}

Supplementary Information accompanies this paper at http://www.nature.com/ naturecommunications

Competing financial interests: The authors declare no competing financial interests.

Reprints and permission information is available online at http://npg.nature.com/ reprintsandpermissions/

How to cite this article: Zheng, $\mathrm{H}$. et al. Tuning the electron transport at single donors in zinc oxide with a scanning tunnelling microscope. Nat. Commun. 4:2992 doi: $10.1038 /$ ncomms3992 (2014) 